\section{MEDICAMENTOS HUÉRFANOS}

Juan Manuel Fontanet Sacristán

Universitat Autònoma de Barcelona / Hospital de la Santa Creu

i Sant Pau (Barcelona)

ORCID iD: https://orcid.org/0000-0003-4170-3776

juanmanuel.fontanet@uab.cat

Josep Torrent-Farnell

Universitat Autònoma de Barcelona / Hospital de la Santa Creu

i Sant Pau (Barcelona)

ORCID iD: https://orcid.org/0000-0003-0620-7507

josep.torrent@uab.cat

Cómo citar este artículo/Citation: Fontanet Sacristán, J. M. Y Torrent-Farnell, J. (2018). Medicamentos huérfanos . Arbor, 194 (789): a466. https://doi.org/10.3989/arbor.2018.789n3008

Recibido: 30 abril 2015. Aceptado: 13 mayo 2016.

RESUMEN: Los medicamentos huérfanos son aquellos fármacos destinados al tratamiento de enfermedades de baja prevalencia, también conocidas como enfermedades raras. La investigación y desarrollo de nuevas terapias para este conjunto heterogéneo de enfermedades presenta una serie de dificultades que están bien reconocidas. Entre ellas, el reducido número de afectados compromete el retorno económico de la inversión realizada por parte de la industria farmacéutica. Por ello la Unión Europea en el año 2000 aprobó un Reglamento Europeo específico para favorecer e incentivar el desarrollo de estas terapias. Transcurrida más de una década, los resultados muestran el efecto positivo que la entrada en vigor de dicho Reglamento ha provocado. Actualmente se han designado más de 1300 compuestos, de los cuales más de 90 ya han obtenido la autorización de comercialización. La voz de los pacientes ha sido clave para el devenir de este cambio y los programas y consorcios promovidos por la UE prometen todavía un futuro más esperanzador.

PALABRAS CLAVE: Enfermedad rara; medicamento huérfano; designación; incentivos; investigación.

\section{ORPHAN DRUGS}

Copyright: (C) 2018 CSIC. Este es un artículo de acceso abierto distribuido bajo los términos de la licencia de uso y distribución Creative Commons Reconocimiento 4.0 Internacional (CC BY 4.0).

ABSTRACT: Orphan drugs are intended for the treatment of diseases of low prevalence, also known as rare diseases. Research and development into new therapies for this heterogeneous group of diseases presents a number of wellrecognized difficulties. One of these is that the small number of patients affected jeopardizes the economic return on the investment made by the pharmaceutical industry. For this reason, in the year 2000 the European Union brought out a specific European Regulation to promote and encourage the development of these therapies. After more than a decade, the results reveal a positive effect of the approval of that regulation. Currently, more than 1300 compounds have been designated orphan drugs, of which over 90 have already obtained marketing authorization. The voice of the patient has been key to producing this change and programs and research consortia promoted by the EU promise an even brighter future.

KEYWORDS: Rare disease; orphan drug; designation; incentives; investigation. 
Las enfermedades raras (ER) o también llamadas enfermedades minoritarias son condiciones patológicas que se presentan de forma poco frecuente en la población afectando a un número reducido de pacientes. Las ER aglutinan a un número enorme y heterogéneo de enfermedades graves y amenazantes de la vida o crónicamente debilitantes que limitan la autonomía y la calidad de vida del afectado y de su entorno familiar. Se han descrito más de 7.000 ER y de estas, unas 5.000 son de base genética y afectan predominantemente a la población pediátrica (Palau, 2010). La prevalencia es el criterio epidemiológico que se utiliza para delimitarlas, y en la Unión Europea la proporción de individuos que debe presentar una enfermedad concreta para ser considerada rara tiene que ser inferior a los cinco casos cada 10.000 habitantes (Regulation (EC) No 141/2000 of the European Parliament and of the Council of 16 December 1999 on orphan medicinal products). El reconocimiento de las necesidades específicas que presentan estos colectivos de afectados fue ya otorgado de forma pionera por el gobierno norteamericano en 1983 (Haffner, 2006). Los medicamentos destinados a tratar enfermedades raras son conocidos como medicamentos huérfanos debido a la falta de estímulos que tradicionalmente venían existiendo para investigar y desarroIlar terapias farmacológicas para este conjunto de enfermedades (Haffner, Torrent-Farnell y Maher, 2008).

En condiciones convencionales el desarrollo de medicamentos es ya en sí mismo un proceso complejo, largo, arriesgado y económicamente costoso que requiere una elevada inversión de recursos y de tiempo, que no siempre finaliza con resultados positivos. Dichas dificultades se ven significativamente incrementadas cuando dichos medicamentos están destinados al tratamiento de enfermedades raras, ya que a los obstáculos propios del procedimiento de desarrollo de fármacos, se unen las limitaciones inherentes de la investigación en enfermedades poco frecuentes (Tambuyzer, 2010). Así pues, factores como la baja prevalencia de la enfermedad, la dificultad en obtener un diagnóstico clínico y genético, la ausencia de registros de pacientes, el limitado número de expertos clínicos en cada una de las enfermedades, la dispersión geográfica de los enfermos, el desconocimiento de la historia natural de la enfermedad, la escasez de estudios epidemiológicos o la ausencia de biomarcadores y variables clínicas validadas, suponen una barrera en la investigación de estas condiciones médicas que complica el desarrollo de nuevas terapias.

Estas dificultades específicas en el desarrollo de medicamentos huérfanos, junto con el hecho de que el potencial número de pacientes que hay que tratar sea bajo o muy bajo (hasta un máximo de 255.550 pacientes en la Unión Europea, o de unos 25.000 pacientes en nuestro país), explica que tradicionalmente el esfuerzo destinado a desarrollar medicamentos dirigidos a tratar enfermedades de baja prevalencia fuera reducido $y$, en consecuencia, hubiera originado una escasez de medicamentos huérfanos. En otras palabras, no existía un entorno de mercado apropiado para que la industria farmacéutica invirtiera en este conjunto tan diverso y complejo de enfermedades.

Ante semejante problemática, en el año 2000 la Unión Europea, siguiendo la experiencia previa adoptada por la Food and Drug Administration (FDA) y los National Institutes of Health (NIH) de Estados Unidos, aprobó el Reglamento Europeo 141/2000 sobre medicamentos huérfanos con el fin de responder al vacío existente en la investigación de fármacos dirigidos a tratar enfermedades raras. Dicho reglamento contemplaba la creación del Comité de Medicamentos Huérfanos (COMP) en el seno de la Agencia Europea del Medicamento (EMA), así como una serie de incentivos para aquellos fármacos en investigación que cumplieran con unos determinados criterios (TorrentFarnell y Morros, 2001).

En este aspecto, el Comité de Medicamentos Huérfanos de la EMA tiene el mandato de evaluar y otorgar la designación de medicamento huérfano (Orphan Designation) a aquellos medicamentos que cumplen con los criterios establecidos para poder ser designados. Los criterios que deben cumplir los fármacos para poder ser designados se resumen en el cuadro 1 , y exigen que dicho medicamento vaya destinado a tratar una enfermedad genuinamente rara o de baja prevalencia y en todo caso inferior a cinco casos por cada 10.000 habitantes en todo el territorio de la Unión Europea, que dicha enfermedad sea amenazante para la vida de los pacientes o crónicamente debilitante y que o bien no existan tratamientos para dicha enfermedad o que, existiendo tales tratamientos, el nuevo medicamento aporte algún beneficio significativo (Significant Benefit) en el abordaje global de la condición que se pretende designar. Aquellos medicamentos en investigación que obtienen la designación huérfana de la EMA/Comisión Europea tienen derecho a incentivos tanto en forma de ventajas económicas y comerciales como de apoyo científico a través de procedimientos específicos (cuadro 2) (Westermark et al., 2011).

Transcurrida más de una década desde la entrada en vigor del Reglamento europeo sobre medicamentos huérfanos, los resultados muestran un significativo 
Cuadro 1. Criterios para la designación de medicamento huérfano en la Unión Europea (Reglamento 141/2000)

- Destinado al diagnóstico, prevención o tratamiento de condiciones amenazantes para la vida o crónicamente debilitantes que afectan a no más de cinco de cada 10.000 personas en la Comunidad Europea cuando la solicitud se realiza (Criterio de prevalencia) o,

- Destinado al diagnóstico, prevención o tratamiento de condiciones amenazantes para la vida o crónicamente debilitantes y que sin incentivos es improbable que la comercialización del medicamento en la Comunidad Europea genere suficiente retorno para justificar la inversión necesaria (Criterio de insuficiente retorno de la inversión) y,

- Que no exista un método satisfactorio de diagnóstico, prevención o tratamiento de la condición en cuestión que haya sido autorizado en la Comunidad Europea (Criterio de método no satisfactorio) o,

- Que en el caso de que exista un método, que el medicamento suponga un beneficio significativo a aquellos afectados por tal condición (Criterio de beneficio significativo).

Fuente: Elaboración propia.

Cuadro 2. Incentivos que aporta la obtención de la designación de medicamentos huérfanos en la Unión Europea.

- Protocol Assistance: acceso al asesoramiento científico por parte de la EMA.

- Procedimiento centralizado: reducción de las tasas para la obtención de la autorización de comercialización de medicamentos huérfanos designados por la Agencia Europea (EMA).

- Exclusividad de mercado: exclusividad de mercado durante diez años que confiere protección contra fármacos similares autorizados en la Unión Europea para la misma indicación terapéutica. Excepciones: acuerdo con el sponsor, falta de suministro, si el nuevo fármaco similar demuestra ser clínicamente superior. Se añaden dos años adicionales si la indicación terapéutica autorizada está destinada a la población pediátrica.

- Incentivos nacionales en los diferentes países de la Unión Europea incluyendo estrategias de apoyo a la I+D+i y políticas de acceso a tales medicamentos por parte de los Sistemas Nacionales de Salud.

- Programas de investigación comunitarios en el marco de la agenda Horizonte 2020 de la Comisión Europea.

Fuente: Elaboración propia.

incremento en la investigación de fármacos dirigidos a tratar, curar o diagnosticar enfermedades consideradas raras. Tanto es así que desde el año 2000, más de 1.300 medicamentos en investigación han sido designados como huérfanos, de los cuales más de 90 medicamentos diferentes han demostrado ser eficaces y seguros por medio de los correspondientes estudios clínicos y han sido autorizados para su comercialización en la Unión Europea (véase tabla 1) (European Medicines Agency. Science Medicines Health. Committee for Orphan Medicinal Products (COMP) meeting report on the review of applications for orphan designation. October 2014).

Merece la pena subrayar el papel complementario y diferenciado que tiene la designación huérfana de la EMA con relación a la autorización de comercialización para una indicación terapéutica huérfana. La primera, como se ha dicho antes, permite un acceso a los medi- camentos solo en el ámbito de la investigación, es decir, a través de programas de investigación y desarrollo clínico mediante ensayos clínicos, o a través de usos compasivos para grupos de pacientes con características similares o para casos individuales excepcionales. Por tanto, la designación huérfana no es intercambiable con la autorización de comercialización; esta última se basa en la evaluación del beneficio y del riesgo potencial y por tanto de la eficacia y de la seguridad del medicamento huérfano para un grupo de afectados que presentan las características clínicas que se especifican en la indicación terapéutica autorizada por la EMA. Debe señalarse a efectos reguladores y jurídicos que la exclusividad de mercado solo protege a la población incluida en la indicación terapéutica aprobada. La designación es por tanto un proceso competitivo (race horse en terminología inglesa) y abierto, en el que diferentes compuestos pueden ser designados para una 
Tabla 1. Estadística de solicitudes, designaciones y medicamentos huérfanos comercializados (abril 2000septiembre 2014)

\begin{tabular}{|c|c|c|c|c|c|}
\hline & $\begin{array}{c}\text { Solicitudes } \\
\text { valoradas }\end{array}$ & $\begin{array}{c}\text { Opiniones } \\
\text { positives } \\
\text { (Designaciones) }\end{array}$ & $\begin{array}{c}\text { Solicitudes } \\
\text { retiradas }\end{array}$ & $\begin{array}{c}\text { Opiniones } \\
\text { negativas }\end{array}$ & $\begin{array}{c}\text { Medicamentos } \\
\text { huérfanos } \\
\text { comercializados }\end{array}$ \\
\hline Total & 2005 & $1352(72 \%)$ & $496(27 \%)$ & $19(1 \%)$ & 96 \\
\hline
\end{tabular}

Fuente: Elaboración propia.

misma enfermedad rara y en el que un mismo principio activo puede ser designado para diferentes condiciones patológicas minoritarias, si se presenta la documentación científica necesaria que avale la plausibilidad del medicamento para cada condición. Finalmente hay que destacar que el proceso de designación europea está abierto tanto a los investigadores como a los centros de investigación académicos, públicos o privados, mientras que el proceso de obtención del registro se limita solo a la industria farmacéutica que deberá cumplir con todos los requisitos que exige la autorización, fabricación y mantenimiento en el mercado de dichas especialidades farmacéuticas.

La contribución de diferentes entidades privadas, públicas, académicas e industriales de nuestro país en impulsar la investigación básica y traslacional en medicamentos huérfanos ha sido notable y creciente, igual que ha sucedido en los principales países de la Unión Europea. Así, 22 promotores españoles han conseguido obtener 40 designaciones de medicamento huérfano, entre los que se incluyen el propio Consejo Superior de Investigaciones Científicas (CSIC) (European Medicines Agency. Science Medicines Health. Public summary of opinion on orphan designation. Raloxifene hydrochloride for the treatment of hereditary haemorrhagic telangiectasia), CIBERER, Almirall, Esteve-UAB, PharmaMar, Digna Biotech, Lipopharma, Tigenix, Proretina Therapeutics, VHIR, Oryzon o Minorix, por citar algunos ejemplos. De entre estos, un producto oncológico (Trabectedina) ha conseguido ya la autorización de comercialización en la Unión Europea.

Resulta indudable, pues, que la creación de un marco regulador específico que incluye ayudas al desarrollo, conjuntamente con la inclusión de las enfermedades raras en los planes de salud pública de los diferentes entes gubernamentales, han supuesto un fuerte impulso en la investigación en medicamentos huérfanos, y por ende en la atención de pacientes y familias afectados por este grupo de patologías.

Este cambio de tendencia iniciado en el año 2000, que ha derivado en un incremento en el interés susci- tado en la investigación en medicamentos huérfanos, se ha visto reforzado por posteriores iniciativas, proyectos, programas y consorcios creados con el mismo fin. En este sentido, la creación de la red Centro Investigación Biomédica en Red en Enfermedades Raras (CIBERER) del Instituto de Salud Carlos III o la realización de telemaratones específicos para financiar proyectos en el ámbito del estado español, la financiación de proyectos cooperativos europeos como los que se enmarcan en el 7th framework o el programa Horizonte 2020 de la Unión Europea, o la fundación del consorcio International Rare Diseases Research Consortium (IRDiRC) en el ámbito internacional son claros ejemplos de proyectos que han contribuido a generar un mayor conocimiento en el campo de las enfermedades raras en general y de los medicamentos huérfanos en particular.

No obstante, si bien el cambio observado y el creciente interés en la investigación en medicamentos huérfanos es muy significativo, siguen existiendo aún hoy ciertas cuestiones en las que se debe seguir ahondando para optimizar tanto la investigación en medicamentos huérfanos como la atención a los pacientes afectos por enfermedades de baja prevalencia.

Por un lado, uno de los retos que se debe afrontar es la adecuación de los ensayos clínicos a las peculiaridades y características específicas propias de las enfermedades raras. Algunos trabajos realizados han puesto de manifiesto que las características de los ensayos clínicos confirmatorios llevados a cabo para demostrar la eficacia de los medicamentos y conseguir la autorización de comercialización para estos medicamentos presentan una menor fortaleza cuando los comparamos con los ensayos clínicos realizados en medicamentos destinados a enfermedades de mayor prevalencia (Buckley, 2008; Califf et al., 2012; Kesselheim, Myers y Avorn, 2011; Mitsumoto, Dorsey, Beck, Kieburtz y Griggs, 2009).

Este hecho ha provocado que en ocasiones se haya puesto en duda la calidad de la evidencia científica que se deriva de alguno de estos estudios (Picavet, Cassiman, Hollak, Maertens y Simoens, 2013). La opti- 
mización de los recursos atendiendo a las características de cada una de las enfermedades raras investigadas es un reto en el que se debe trabajar con firmeza. En este aspecto, parece necesario ajustar los diseños metodológicos convencionales a la realidad de estas enfermedades de un modo flexible, así como propiciar la utilización de diseños metodológicos alternativos que permitan un mayor aprovechamiento de los recursos disponibles en cada caso particular (Bowalekar, 2011; Gupta, Faughnan, Tomlinson y Bayoumi, 2011). Por ello se han iniciado proyectos promovidos por la Unión Europea que actualmente se están desarrollando, en los que se trabaja para conseguir una mejora en la investigación en enfermedades raras y medicamentos huérfanos siempre preservando los pilares básicos de la investigación: calidad, eficacia y seguridad (Tudur Smith, Williamson y Beresford, 2014).

Por otra parte, no se puede obviar el elevado nivel de controversia en cuanto a las posibilidades de acceso de los pacientes a los diferentes tratamientos farmacológicos, debido al elevado precio que se otorga a los medicamentos huérfanos para compensar el bajo número de pacientes que hay que tratar y que supone un elevado impacto presupuestario en los diferentes sistemas sanitarios estatales y autonómicos. Este hecho junto con la desfavorable coyuntura económica puede ocasionar dificultades en el acceso a los medicamentos huérfanos de los pacientes afectados (Hughes-Wilson, Palma, Schuurman y Simoens, 2012; Simoens, 2011).
El desarrollo de políticas sanitarias que favorezcan la equidad en el acceso a fármacos para enfermedades raras es crucial para favorecer la sostenibilidad del sistema sanitario, evitando poner trabas a la innovación que aporte valor añadido en el tratamiento de estos pacientes (Morel et al., 2013).

En definitiva, la investigación y el desarrollo en medicamentos huérfanos han experimentado un innegable auge en los últimos años que debe traducirse en una mejora en la calidad de vida de los pacientes afectados por enfermedades raras, así como de sus familiares y cuidadores. Adicionalmente este avance debe repercutir también en la investigación de otras enfermedades convencionales ya que, debido al carácter transversal del conocimiento médico, la experiencia adquirida en las enfermedades raras puede potencialmente ser de aplicación en patologías comunes, aportando ventajas para el conjunto de la sociedad. Finalmente debe reconocerse el papel activo y crucial que las asociaciones de pacientes han desarrollado a lo largo del camino recorrido tanto en Estados Unidos como en los diferentes estados de la Unión Europea. Asociaciones de pacientes afectos por enfermedades raras como EURORDIS en el ámbito europeo o FEDER en el español han sido catalizadores del auge en la investigación en medicamentos huérfanos, impulsando la formación y educación y las políticas de transparencia y acceso a estas intervenciones terapéuticas.

\section{BIBLIOGRAFÍA}

Bowalekar, S. (2011). Adaptive designs in clinical trials. Perspectives in Clinical Research, 2 (1), pp. 23-27. https://doi. org/10.4103/2229-3485.76286

Buckley, B. M. (2008). Clinical trials of orphan medicines. Lancet, 371 (9629), pp. 2051-2055. https://doi. org/10.1016/S0140-6736(08)60876-4

Califf, R. M., Zarin, D. A., Kramer, J. M., Sherman, R. E., Aberle, L. H. y Tasneem, A. (2012). Characteristics of clinical trials registered in ClinicalTrials. gov, 2007-2010. Jama 307 (17), pp. 1838-1847. https://doi.org/10.1001/ jama.2012.3424

Gupta, S., Faughnan, M. E., Tomlinson, G. A. y Bayoumi, A. M. (2011). A framework for applying unfamiliar trial designs in studies of rare diseases. Journal of Clinical Epidemiology, 64 (10), pp. 1085-
1094. https://doi.org/10.1016/j.jclinepi.2010.12.019

Haffner, M. E. (2006). Adopting Orphan Drugs - Two Dozen Years of Treating Rare Diseases. New England Journal of Medicine, 354 (5), pp. 445-447. https:// doi.org/10.1056/NEJMp058317

Haffner, M. E., Torrent-Farnell, J. y Maher, P. D. (2008). Does orphan drug legislation really answer the needs of patients? The lancet, 371 (9629), pp. 20412044. https://doi.org/10.1016/S01406736(08)60873-9

Hughes-Wilson, W., Palma, A., Schuurman, A. y Simoens, S. (2012). Paying for the Orphan Drug System: break or bend? Is it time for a new evaluation system for payers in Europe to take account of new rare disease treatments? Orphanet Journal of
Rare Diseases, 7 (1), 74. https://doi. org/10.1186/1750-1172-7-74

Kesselheim, A. S., Myers, J. A. y Avorn, J. (2011). Characteristics of clinical trials to support approval of orphan vs nonorphan drugs for cancer. JAMA. The Journal of American Medical Association, 305 (22), pp. 2320-2326. https:// doi.org/10.1001/jama.2011.769

Mitsumoto, J., Dorsey, E. R., Beck, C. A., Kieburtz, K. y Griggs, R. C. (2009). Pivotal studies of orphan drugs approved for neurological diseases. Annals of Neurology. Official Journal of the American Neurological Association and the Chikd Neurology Society, 66 (2), pp. 184-190.

Morel, T., Arickx, F., Befrits, G., Siviero, P., Meijden, C. van der, Xoxi, E. y Simoens, S. (2013). Reconciling uncertainty of costs and outcomes with the need for 
access to orphan medicinal products: a comparative study of managed entry agreements across seven European countries. Orphanet Journal of Rare Diseases, 8 (1), 198. https://doi. org/10.1186/1750-1172-8-198

Palau, F. (2010). Enfermedades raras, un paradigma emergente en la medicina del siglo XXI. Medicina Clínica, 134 (4), pp. 161-168. https://doi.org/10.1016/j. medcli.2009.06.038

Picavet, E., Cassiman, D., Hollak, C. E., Maertens, J. A. y Simoens, S. (2013). Clinical evidence for orphan medicinal products-a cause for concern? Orphanet Journal of Rare Diseases, 8 (1), 164. https://doi.org/10.1186/17501172-8-164

Simoens, S. (2011). Pricing and reimbursement of orphan drugs: the need for more transparency. Orphanet Journal of Rare Diseases, 6 (1), 42. https://doi. org/10.1186/1750-1172-6-42

Tambuyzer, E. (2010). Rare diseases, orphan drugs and their regulation: questions and misconceptions. Nature Reviews.
Drug Discovery, 9 (12), pp. 921-929. https://doi.org/10.1038/nrd3275

Torrent-Farnell, J. y Morros, R. (2001). The $\mathrm{EU}$ challenges on the designation of orphan medicinal products. Pharmaceuticals Policy and Law, 3, pp. 19-30.

Tudur Smith, C., Williamson, P. R. y Beresford, M. W. (2014). Methodology of clinical trials for rare diseases. Best Practice \& Research. Clinical Rheumatology, 28 (2), pp. 247-262. https://doi. org/10.1016/j.berh.2014.03.004

Westermark, K., Holm, B. B., Söderholm, M., Llinares-García, J., Rivière, F., Aarum, S. [...] y Belorgee, C. (2011). European regulation on orphan medicinal products: 10 years of experience and future perspectives. Nature Reviews. Drug Discovery, 10 (5), pp. 341-349. https:// doi.org/10.1038/nrd3445

\section{Recursos en línea}

European Medicines Agency. Science Medicines Health. Committee for Orphan Medicinal Products (COMP) meeting report on the review of applications for orphan designation. October 2014. [En línea]. Disponible en https://www.ema. europa.eu/documents/committeereport/comp-meeting-report-reviewapplications-orphan-designation-october-2014_en.pdf

European Medicines Agency. Science Medicines Health. Public summary of opinion on orphan designation. Raloxifene hydrochloride for the treatment of hereditary haemorrhagic telangiectasia. [En línea]. Disponible en: https:// www.ema.europa.eu/documents/ orphan-designation/eu/3/10/730public-summary-opinion-orphandesignation-raloxifene-hydrochloridetreatment-hereditary_en.pdf

Regulation (EC) No 141/2000 of the European Parliament and of the Council of 16 December 1999 on orphan medicinal products. Official Journal of the European Communities, L18, pp. 1-5. [En línea]. Disponible en https://ec.europa. eu/health/sites/health/files/files/ eudralex/vol-1/reg_2000_141_cons2009-07/reg_2000_141_cons-200907_en.pdf 